Check for updates

Cite this: RSC Adv., 2018, 8, 42073

\title{
Influence of atmospheric species on the electrical properties of functionalized graphene sheets
}

\author{
Bilge Bekdüz, (D) a Laura Kampermann, (D) a Wolfgang Mertin, (D) *a Christian Punckt, $\uparrow^{\text {b }}$ \\ Ilhan A. Aksay ${ }^{\mathrm{b}}$ and Gerd Bacher (iD ${ }^{\mathrm{a}}$
}

We report on the time-dependent influence of atmospheric species on the electrical properties of functionalized graphene sheets (FGSs). When exposed to laboratory air, FGSs exhibit a significant, irreversible decrease in electrical conductance with time, strongly depending on the oxygen content of the FGSs. To separate the roles of charge carrier density and mobility in this aging process, we performed electron transport measurements using a back-gate field-effect transistor architecture. Investigating the position of the Dirac point under different atmospheres, we found that adsorbed atmospheric species result in pronounced p-doping, which - on a short time scale - can be reversed under nitrogen atmosphere. However, on a time scale of several days, the resistance increases irreversibly, while the Dirac point voltage remains constant. From these experiments, we conclude that the aging of FGSS is related to the chemisorption of atmospheric species leading to enhanced carrier scattering due to an increasing amount of $\mathrm{sp}^{3}$ - regions and thus to a reduced charge carrier mobility.

Received 4th October 2018

Accepted 10th December 2018

DOI: $10.1039 / \mathrm{c} 8 \mathrm{ra0} 8227 \mathrm{~h}$

rsc.li/rsc-advances

(FGSs). During thermal exfoliation and reduction, the material is not completely reduced, but one instead obtains a graphenelike material with a high number of lattice defects and oxygencontaining functional groups (Fig. 1). The ratio of the molar content of carbon and oxygen in the material, the $\mathrm{C} / \mathrm{O}$ ratio $x$, can be used as a measure for the degree of reduction.

While the chemical route produces a material with a lower number density of lattice defects, it is less effective than the thermal route in reducing the material. It is known that chemically reduced rGO typically exhibits an $\mathrm{O} / \mathrm{C}$ ratio above $4.19 \%$, corresponding to a C/O ratio $x<24 .{ }^{21}$ Directly exfoliated graphite - while showing a small level of defectiveness -

There are different routes for obtaining graphene or graphene-like materials in bulk quantities, including exfoliation and reduction of graphite oxide (GO) via chemical $^{\mathbf{1 6}}$ or thermal $^{17}$ means, or the direct exfoliation of graphite, ${ }^{18}$ each yielding materials with different chemical and electrical properties depending on the synthesis route and parameters. ${ }^{\mathbf{1 9 2 0}}$

In the chemical route, the GO is first separated into graphene oxide sheets via dispersion in a suitable solvent and subsequently reduced chemically, yielding a material we will refer to as reduced graphene oxide (rGO). In case of the thermal exfoliation process, sheets are separated and reduced simultaneously, yielding so-called functionalized graphene sheets

${ }^{a}$ Werkstoffe der Elektrotechnik, CENIDE, Universität Duisburg-Essen, 47057 Duisburg, Germany.E-mail:wolfgang.mertin@uni-due.de

${ }^{b}$ Department of Chemical and Biological Engineering, Princeton University, Princeton, New Jersey 08544, USA

$\dagger$ Present address: Institute of Nanotechnology, Karlsruhe Institute of Technology, 76344 Eggenstein-Leopoldshafen, Germany.
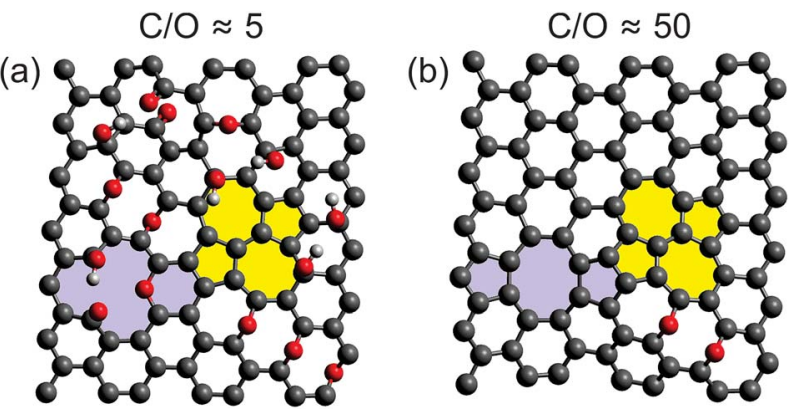

Fig. 1 Schematic representation of functionalized graphene at different degrees of reduction as indicated. Besides the functionalization of the basal plane of graphene through epoxy and hydroxyl groups as well as ketones, also a divacancy (light violet) and a StoneWales defect (yellow) are shown as representative examples of lattice defects. The red dots indicate oxygen and light grey ones hydrogen atoms. 
contains almost no oxygen $(x \rightarrow \infty)$. FGS, however, can be tuned from a $\mathrm{C} / \mathrm{O}$ ratio of $x=2$ (no reduction) to values in excess of 500, thus approaching pristine graphene. Since the degree of thermal reduction determines the material's electrical properties, FGSs offer the unique possibility of tuning the electrical conductivity of the material from insulating over semiconducting to conducting. ${ }^{19,20}$

However, for commercial applications of FGSs, the long-term stability of performance-determining material properties is essential. In particular in applications, where FGSs are exposed to the environment, e.g., to oxygen and humidity from the air, it is crucial to understand, how the environment may affect the electrical properties of FGSs and thus of the whole material system into which the FGSs have been incorporated.

Investigations of the effect of environment on the electronic properties of graphene have focused on mechanically exfoliated $^{22,23}$ and chemical vapor-deposited (CVD) ${ }^{24-29}$ graphene. For example, Balandin's group investigated the electrical and noise characteristics of a field-effect transistor structure based of mechanically exfoliated graphene, when it was exposed to ambient conditions for over a month..$^{22,23}$ They found an increase in noise with time, which was attributed to a decrease in carrier mobility and an increase of the contact resistances. For CVD graphene, Yavari et al. observed a saturation behaviour in the change of the resistance, when the graphene was exposed to humid air for 7.5 hours, and the time to reach the saturation dependent on the absolute humidity. ${ }^{24}$ In contrast, Pustelny et al. did not observe any saturation within 4 hours. ${ }^{26}$ A number of recent investigations discussed the stability of n- or p-doped CVD graphene. Xu et al. doped CVD graphene via adsorption of an organic compound (n type) and found stable values of the carrier mobility in air for up to 1000 hours. ${ }^{27}$ Using thermal treatment at $300{ }^{\circ} \mathrm{C}$ in an oxygen atmosphere, Piazza et al. obtained p-doping of graphene, which was stable even after a thermal treatment for up to 15 hours. ${ }^{28}$ Melios et al. showed that mono and bi-layer CVD graphene is extremely sensitive to water substances in controlled humidity environments. ${ }^{29}$

Much less is known about the influence of ambient atmosphere on the electrical properties of $\mathrm{rGO}^{30-33}$ or even FGS. Gómez-Navarro et al. found a significant shift of the Dirac-point to positive gate-source voltages $V_{\mathrm{GS}}$, when rGO was exposed to air atmosphere for 24 hours, which could be reversed by placing the graphene back in vacuum. ${ }^{30}$ Gupta et al. showed that during aging of polyethylene glycol-rGO composites for 3-6 months the number of oxygen-containing functional groups increases with time. ${ }^{33}$ Most important, no systematic studies of adsorption effects exist in case of FGSs (although expected to be similar to those obtained with rGO) and irreversible effects - leading to aging - have been largely neglected both in rGO and FGS.

In this work, we systematically analyze the long-term stability of the electronic properties of FGSs with different degrees of functionalization, using materials with $\mathrm{C} / \mathrm{O}$ ratios between $x=$ 5.4 and $x=60$ under air and nitrogen atmosphere. Using a back-gate field effect transistor configuration, the doping level and the charge carrier mobility for FGSs can be determined separately. Our experiments give access to the impact of physisorbed and chemisorbed atmospheric species on the electronic properties of FGSs.

\section{Experimental section}

\section{Preparation of FGSs}

FGSs were prepared by thermal exfoliation of graphite oxide, as described in detail elsewhere. ${ }^{17,20}$ In short, individual graphene sheets are separated due to the gas evolution at high temperatures. Hereby the $\mathrm{C} / \mathrm{O}$ ratio increases simultaneously due to the removal of oxygen. ${ }^{17}$ Depending on the temperature and the duration time of the exfoliation process, $\mathrm{C} / \mathrm{O}$ ratios can be varied between 4 and $340 .^{19,20}$ The samples used in this work were prepared with the following parameters: $300{ }^{\circ} \mathrm{C}$ for $1 \mathrm{~min}$ resulting in a $\mathrm{C} / \mathrm{O}$ ratio of $5.4 \pm 0.2,500{ }^{\circ} \mathrm{C}$ for $1 \mathrm{~min}(\mathrm{C} / \mathrm{O}=6 \pm$ $0.3), 1100{ }^{\circ} \mathrm{C}$ for $1 \mathrm{~min}(\mathrm{C} / \mathrm{O}=26 \pm 2)$ and $1100^{\circ} \mathrm{C}$ for $10 \mathrm{~min}(\mathrm{C} /$ $\mathrm{O}=60 \pm 12$ ).

\section{Device fabrication}

To evaluate the electrical properties of a single FGS a standard back-gated field-effect transistor device architecture was used. ${ }^{2}$ Prior to processing, the FGS powders are stored under nitrogen atmosphere. For electrically contacting individual graphene sheets with typical sizes in the sub- $\mu \mathrm{m}$ range, a highly n-doped Si substrate with an insulating $90 \mathrm{~nm} \mathrm{SiO}$ layer on top was prepatterned with alignment marks $(10 \mathrm{~nm} / 50 \mathrm{~nm} \mathrm{Ti} / \mathrm{Au})$ via electron beam lithography (Zeiss Supra 25) in order to subsequently define the positions of the sheets to be contacted relative to the marks. The FGSs were dispersed in ethanol (C/O ratios of 5.4, 6, and 26) or 1,2-dichloroethane (C/O ratio of 60), tip-sonicated (Bandelin Sonopuls HD 2070) at $21 \mathrm{~W}$ power centrifuged at $1073 g$ for an hour each. Then the dispersion was drop-cast onto the pre-patterned substrate and dried at room temperature. After defining the positions with the help of the alignment marks, the contacts were designed by an Elphy Quantum lithography tool and patterned via electron beam lithography using $310 \mathrm{~nm}$ PMMA as a resist, which after exposure was developed with methyl isobutyl ketone (1 minute) and 2-propanol (1 minute) and blown dried under a nitrogen stream. $10 \mathrm{~nm}$ of $\mathrm{Ti}$ as an adhesion layer and $50 \mathrm{~nm}$ of Au was evaporated afterwards as contact materials (Oerlikon Leybold Vacuum Univex 350). The lift-off process was performed by leaving the sample in an acetone bath overnight and subsequent, gentle rinsing with 2-propanol, followed by blow-drying under a stream of nitrogen.

\section{Measurement and analysis procedure}

The electrical behavior of a single FGS can be characterized by the electrical current flowing from the drain to the source contact $\left(I_{\mathrm{DS}}\right)$ in dependence of a drain-source voltage $\left(V_{\mathrm{DS}}\right)$ and a gate-source voltage $\left(V_{\mathrm{GS}}\right){ }^{2}{ }^{2}$ These measurements $\left(I_{\mathrm{DS}} v s . V_{\mathrm{DS}}\right.$ resp. $\left.V_{\mathrm{GS}}\right)$ were conducted by applying a drain-source voltage $V_{\text {DS }}$ (Yokogawa GS 200) and measuring the drain-source current $I_{\mathrm{DS}}$ with a current-to-voltage converter (Femto DLPCA-200) and a multimeter (Keithley 2000). To avoid damages to the FGSs and to exclude artifacts due to different applied currents in case of 
different $\mathrm{C} / \mathrm{O}$ ratios, $V_{\mathrm{DS}}$ was varied for each sample such that $I_{\mathrm{DS}}$ did not exceed $150 \mathrm{nA}$. For each $\mathrm{C} / \mathrm{O}$ ratio, the resistance was measured for 3 to 7 samples, and an average value and a standard deviation were calculated. To study the effect of aging, the $I_{\mathrm{DS}} / V_{\mathrm{DS}}$ measurements were repeated each day for a period of about a week. Between the measurements, the samples were stored under laboratory air in a clean room (humidity of $40 \%$ at $20.5{ }^{\circ} \mathrm{C}$ ). The measurements were conducted either under atmospheric conditions or under continuous nitrogen flow. The nitrogen flow was activated at least an hour prior to measurement, allowing the system to equilibrate. In addition, for the reference measurement the test specimens were kept under nitrogen flow for the whole duration of the long-term measurement.

The gate-source voltage $V_{\mathrm{GS}}$ was applied by a source meter (Keithley 2601A) through a copper foil attached to the backside of the silicon substrate. The field-effect dependence of the resistance was measured as a function of $V_{\mathrm{GS}}$. From the slope of the $I_{\mathrm{DS}} v s$. $V_{\mathrm{GS}}$ curve the mobilities of the charge carriers are extracted using the equation ${ }^{34}$

$$
\mu=\frac{\partial I_{\mathrm{DS}}}{\partial V_{\mathrm{GS}}} \frac{L \times d}{b \times \varepsilon_{0} \times \varepsilon_{\mathrm{r}} \times V_{\mathrm{DS}}}
$$

where $L$ is the length and $b$ is the width of the graphene channel between drain and source contacts, extracted as described elsewhere. ${ }^{20} d$ is the thickness of the $\mathrm{SiO}_{2}$ layer (in our case 90 $\mathrm{nm}), \varepsilon_{0}$ is the permittivity of vacuum and $\varepsilon_{\mathrm{r}}$ is the relative permittivity of $\mathrm{SiO}_{2}$ (assumed as 3.9). To get a reliable comparison between the mobilities extracted from different data sets, we analyzed the slope of the $I_{\mathrm{DS}} v s$. $V_{\mathrm{GS}}$ curve at a welldefined Fermi level shift $\left(\left|\Delta E_{\mathrm{F}}\right|=0.15 \mathrm{eV}\right.$ corresponding to a charge carrier density of $1.4 \times 10^{12} \mathrm{~cm}^{-2}$ ) relative to the Dirac point voltage $\left(V_{\mathrm{GSO}}\right)$ as given by ${ }^{35}$

$$
\Delta E_{\mathrm{F}}= \pm \hbar \times \nu_{\mathrm{F}} \times \sqrt{\pi \times n_{\mathrm{c}}}
$$

where $n_{\mathrm{c}}$ is obtained from ${ }^{36}$

$$
n_{\mathrm{c}}=\frac{1}{e} \frac{\left|V_{\mathrm{GS}}-V_{\mathrm{GSO}}\right| \times \varepsilon_{0} \times \varepsilon_{\mathrm{r}}}{d}
$$

Here, $e$ is the elementary charge, $\hbar$ the reduced Planck constant, and $\nu_{\mathrm{F}}=1.1 \times 10^{6} \mathrm{~m} \mathrm{~s}^{-1}$ the Fermi velocity. ${ }^{37}$

\section{Results and discussion}

The details about preparation methods and the measurement and analysis procedures are provided in the Experimental section. The samples with varied $\mathrm{C} / \mathrm{O}$ ratio, $x$ ranging from 5.4 to 60 were prepared as described in ref. 17 and 20 . The C/O ratios were calculated from EDX measurements on pellets that are fabricated from powders with different exfoliation parameters and have been confirmed by CHNO analysis, giving a $1: 1$ correlation. Exfoliation parameters and the resulting $\mathrm{C} / \mathrm{O}$ ratios are shown in Table 1. Indicated errors represent the standard deviation obtained from measurements at different locations on pressed FGS powder pellets.

The $I_{\mathrm{DS}} / V_{\mathrm{DS}}$ curves obtained for FGS with C/O ratios of $x=5.4$ and $x=60$ measured on different days under atmospheric
Table 1 Exfoliation parameters and resulting $\mathrm{C} / \mathrm{O}$ ratios of the materials used in this work

\begin{tabular}{lcc}
\hline Exfoliation & C/O ratio $x$ & $\begin{array}{l}\text { Oxygen content } \\
\text { in at\% }\end{array}$ \\
\hline $300{ }^{\circ} \mathrm{C}, 60 \mathrm{~s}$ & $5.4 \pm 0.2$ & $15.6 \pm 0.6$ \\
$500{ }^{\circ} \mathrm{C}, 60 \mathrm{~s}$ & $6 \pm 0.3$ & $14.3 \pm 0.7$ \\
$1100{ }^{\circ} \mathrm{C}, 60 \mathrm{~s}$ & $26 \pm 2$ & $3.7 \pm 0.3$ \\
$1100^{\circ} \mathrm{C}, 600 \mathrm{~s}$ & $60 \pm 12$ & $1.6 \pm 0.3$ \\
\hline
\end{tabular}

environment and under nitrogen flow are shown in Fig. 2. The measurements were performed within identical current ranges for samples of different $\mathrm{C} / \mathrm{O}$ ratios to ensure that observed differences are not caused by current-related artefacts. A nonlinear behavior is apparent for $x=5.4$ both under air [Fig. 2(a), top] and under nitrogen [Fig. 1(a), bottom] conditions, whereas the sample with a high $\mathrm{C} / \mathrm{O}$ ratio of $x=60$ shows a linear $I_{\mathrm{DS}} / V_{\mathrm{DS}}$ dependence. ${ }^{20} I / V$ curves for intermediate values of $x$ fall in between those shown in Fig. 2.

The change from non-linear to linear $I_{\mathrm{DS}} / V_{\mathrm{DS}}$ behavior for low to high $\mathrm{C} / \mathrm{O}$ ratios is comparable to what is reported in the literature and can be attributed to an increase of $\mathrm{sp}^{2}$-hybridized islands in a $\mathrm{sp}^{3}$-hybridized backbone with decreasing oxidation resulting in a change of the transport mechanism from mainly hopping to diffusive. ${ }^{20,30,38}$ For low $\mathrm{C} / \mathrm{O}$ ratios the contacts might in addition contribute to the overall resistance. ${ }^{20}$ At a first glance, only a small variation of the $I / V$ characteristics with atmosphere is found for both $\mathrm{C} / \mathrm{O}$ ratios. Independent of the $\mathrm{C} /$ $O$ ratio $x$, however, a pronounced decrease in the current at any given $V_{\mathrm{DS}}$ is observed, when the measurements are repeated over a period of a few days.

Due to the partly non-linear nature of the current-voltage relation, we calculated the differential conductance at different current values $(0,-100 \mathrm{nA},+90 \mathrm{nA})$. These data are normalized to the differential conductance measured on the first day, which
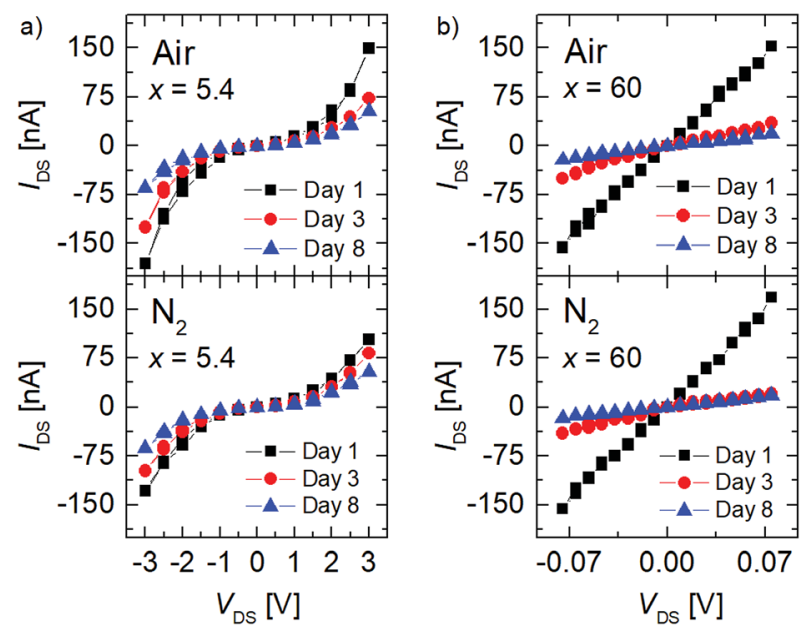

Fig. $2 I_{D S} / V_{D S}$ characteristics of selected single FGS transistor devices for $\mathrm{C} / O$ ratios (a) $x=5.4$ and (b) $x=60$ under air (top) and $\mathrm{N}_{2}$ (bottom) atmosphere at different days. The gate of the device was left unconnected (floating gate). 

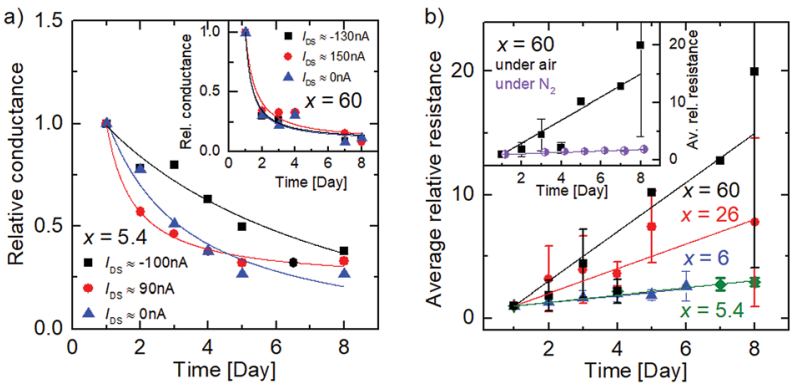

Fig. 3 Time dependent aging of the FGSs (a) relative conductance $v$ s. time for FGSs with C/O ratios of $x=5.4$ and $x=60$ (inset) (b) average relative resistance for different values $x$ as a function of time under air. The inset compares data where the sample $(x=60)$ was stored in air or under nitrogen atmosphere, respectively, for the whole time.

is shown in Fig. 3(a) as a function of time. A decrease in the relative differential conductance is observed with aging time in all current regimes for $x=5.4$ and $x=60$ (inset), indicating that the aging is independent of the non-linearity of the $I / V$ curves.

To discuss the $\mathrm{C} / \mathrm{O}$ ratio-dependent aging, we calculated the relative differential resistance around $I_{\mathrm{DS}} \approx 0$ A for different samples and plotted the average values as a function of time in Fig. 3(b). A clear increase in the relative resistance is shown due to aging, becoming more prominent as the $\mathrm{C} / \mathrm{O}$ ratio increases. The resistance changes by about a factor of three for $x=5.4$, whereas the resistance for $x=60$ increases by more than an order of magnitude from day 1 to day 8 . The resistance increase of the $x=6$ sample is comparable to that observed for $x=5.4$, and the value for $x=26$ lies between both extrema. Thus, we observe an increase of resistance over time, whose relative amount increases with $\mathrm{C} / \mathrm{O}$ ratio. Reference measurements, where the sample was strictly kept under nitrogen atmosphere for the whole time, were performed for $x=60$ and can be seen in the inset of Fig. 3(b). The increase in resistance is negligible compared to the alteration under laboratory air indicating that the aging is caused solely by the atmospheric species. We attribute our observations to an aging of the FGSs rather than to a change of the metal/graphene contacts, because a contact resistance change, e.g., due to the oxidation of the $\mathrm{Ti} / \mathrm{Au}$ contacts, should be virtually independent of the $\mathrm{C} / \mathrm{O}$ ratio.

Since the resistance of the FGS sheets depends on both the charge carrier density and the carrier mobility, it is necessary to figure out the role of both during aging. For that purpose, we measured the $I_{\mathrm{DS}} / V_{\mathrm{GS}}$ characteristic with varying back-gate voltages. By applying a back-gate voltage, the charge carrier density in the FGS changes and therefore the Fermi level shifts. Negative (positive) voltages induce holes (electrons) and shift the Fermi level accordingly. ${ }^{39}$

To study the atmospheric doping effect of FGSs with different $\mathrm{C} / \mathrm{O}$ ratios, we performed measurements with varying gate-source voltages $V_{\mathrm{GS}}$ under different environmental conditions and the results are shown in Fig. 4. Increasing the amount of functional groups, i.e., decreasing the $\mathrm{C} / \mathrm{O}$ ratio $x$, the overall resistance increases both under air [Fig. 4(a)] and nitrogen atmosphere [Fig. 4(b)]. This trend agrees well with literature. ${ }^{20}$ We measure a Dirac point voltage of $V_{\mathrm{GSO}} \geq 25 \mathrm{~V}$ under ambient
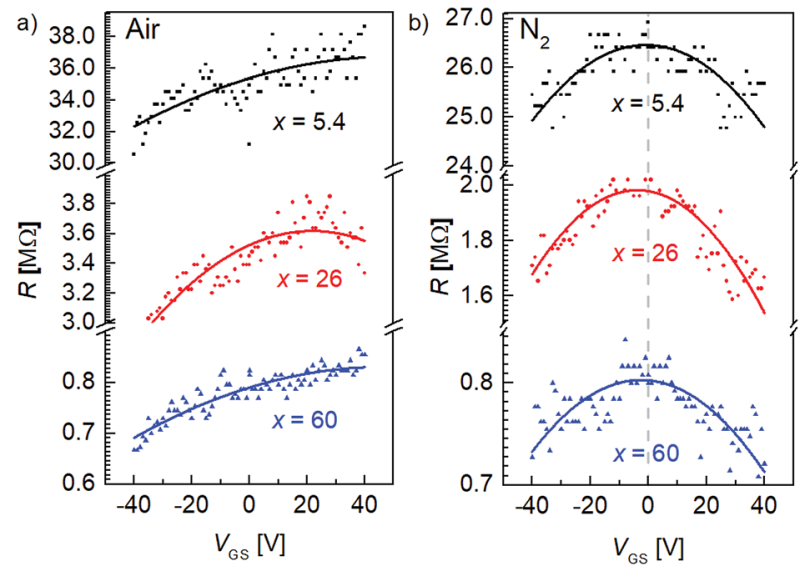

Fig. 4 Electrical field-effect for FGSs with different C/O-ratios measured (a) under air and (b) under nitrogen atmosphere.

conditions for all $\mathrm{C} / \mathrm{O}$ ratios, indicating pronounced p-type doping for all types of FGSs. In contrast, $V_{\mathrm{GSo}}$ is shifted to about $0 \mathrm{~V}$ under $\mathrm{N}_{2}$ atmosphere. A similar behavior has been also reported in the literature for $\mathrm{rGO}^{30}$ where it has been shown that under vacuum $V_{\mathrm{GSo}}$ is shifted close to zero. Within our experimental error, the back-gate voltage dependence under $\mathrm{N}_{2}$ atmosphere is almost symmetric for $\mathrm{p}$ - and $\mathrm{n}$-type transport regimes.

In contrast to pristine graphene, a parabolic behavior is observed in rGO and FGS close to Dirac point voltage rather than a linear relationship ${ }^{30,31}$ due to the large amount of scattering. We thus defined a fixed value of the charge carrier density (i.e., a fixed Fermi level, see Experimental section), in order to compare the mobility values of FGSs with varying $\mathrm{C} / \mathrm{O}$ ratios and emphasize that the extracted values for charge carrier mobility should be understood as first approximations. We extracted the hole mobility from the data depicted in Fig. 4 at a Fermi level shift of $\Delta E_{\mathrm{F}}=-0.15 \mathrm{eV}$ into the valence band, where we have a p-type transport, and the electron mobility at a value of $\Delta E_{\mathrm{F}}=0.15 \mathrm{eV}$ into the conduction band, yielding a ntype transport. The values obtained for the field-effect mobility for FGSs with different $\mathrm{C} / \mathrm{O}$ ratios are extracted and summarized in Table 2 for ambient conditions and for nitrogen atmosphere. Due to high p-type doping under air the mobility of the electrons is not accessible. By decreasing the amount of oxygen from $x=5.4$ to $x=60$, the hole mobility is found to increase by roughly two orders of magnitude under ambient conditions. We obtain hole mobilities of $\mu_{\mathrm{p}}=0.16 \times 10^{-3} \mathrm{~cm}^{2} \mathrm{~V}^{-1} \mathrm{~s}^{-1}$ for $x=$ 5.4 and $\mu_{\mathrm{p}}=11 \times 10^{-3} \mathrm{~cm}^{2} \mathrm{~V}^{-1} \mathrm{~s}^{-1}$ for $x=60$. These values are in reasonable agreement with literature data found for rGO, where values of $0.01 \mathrm{~cm}^{2} \mathrm{~V}^{-1} \mathrm{~s}^{-1}$ to $1 \mathrm{~cm}^{2} \mathrm{~V}^{-1} \mathrm{~s}^{-1}$ are reported. $^{38,40}$ The same trend for the hole mobility is apparent under nitrogen atmosphere, where the electron mobility can be extracted additionally. An electron mobility of $\mu_{\mathrm{n}}=0.06 \times 10^{-3}$ $\mathrm{cm}^{2} \mathrm{~V}^{-1} \mathrm{~s}^{-1}$ is obtained for $x=5.4$, increasing by a factor of 200 for $x=60$.

The measurement of the back-gate voltage dependence was repeated at different days for FGSs with a C/O ratio of $x=26$ under air and nitrogen. From Fig. 5 it can be seen, that although 
Table 2 Charge carrier mobility under air (only holes) and nitrogen (holes and electrons) for different C/O ratios, when the Fermi level shift reaches the values of $-0.15 \mathrm{eV}$ (holes) and $+0.15 \mathrm{eV}$ (electrons), respectively

\begin{tabular}{|c|c|c|c|}
\hline \multirow[b]{2}{*}{$\mathrm{C} / \mathrm{O}$ ratio, $x$} & \multirow{2}{*}{$\begin{array}{l}\text { Air [day 2] } \\
\mu_{\mathrm{p}} 10^{3}\left[\mathrm{~cm}^{2} \mathrm{~V}^{-1} \mathrm{~s}^{-1}\right]\end{array}$} & \multicolumn{2}{|l|}{ Nitrogen [day 3] } \\
\hline & & $\mu_{\mathrm{p}} 10^{3}\left[\mathrm{~cm}^{2} \mathrm{~V}^{-1} \mathrm{~s}^{-1}\right]$ & $\mu_{\mathrm{n}} 10^{3}\left[\mathrm{~cm}^{2} \mathrm{~V}^{-1} \mathrm{~s}^{-1}\right]$ \\
\hline 5.4 & 0.16 & 0.06 & 0.06 \\
\hline 26 & 4 & 2.2 & 2.9 \\
\hline 60 & 11 & 9 & 12 \\
\hline
\end{tabular}

a slight shift of the Dirac point voltage seems to occur under ambient atmosphere, the sample remains strongly p-doped after aging under ambient conditions. In addition, no shift of the Dirac point voltage is seen when the samples are measured under nitrogen atmosphere after different days of aging. This indicates that the Fermi energy changes only weakly as a result of the aging process.

We thus conclude that the observed increase in resistance over the course of several days is caused by a decreasing mobility during aging. Agreeing with Gupta $e t$ al. ${ }^{33}$ the amount of functional groups and hence the amount of $\mathrm{sp}^{3}$-hybridization might increase during aging, resulting in an increased carrier scattering, limiting the electronic transport. We attribute this to incorporation (chemisorption) of adsorbing atmospheric

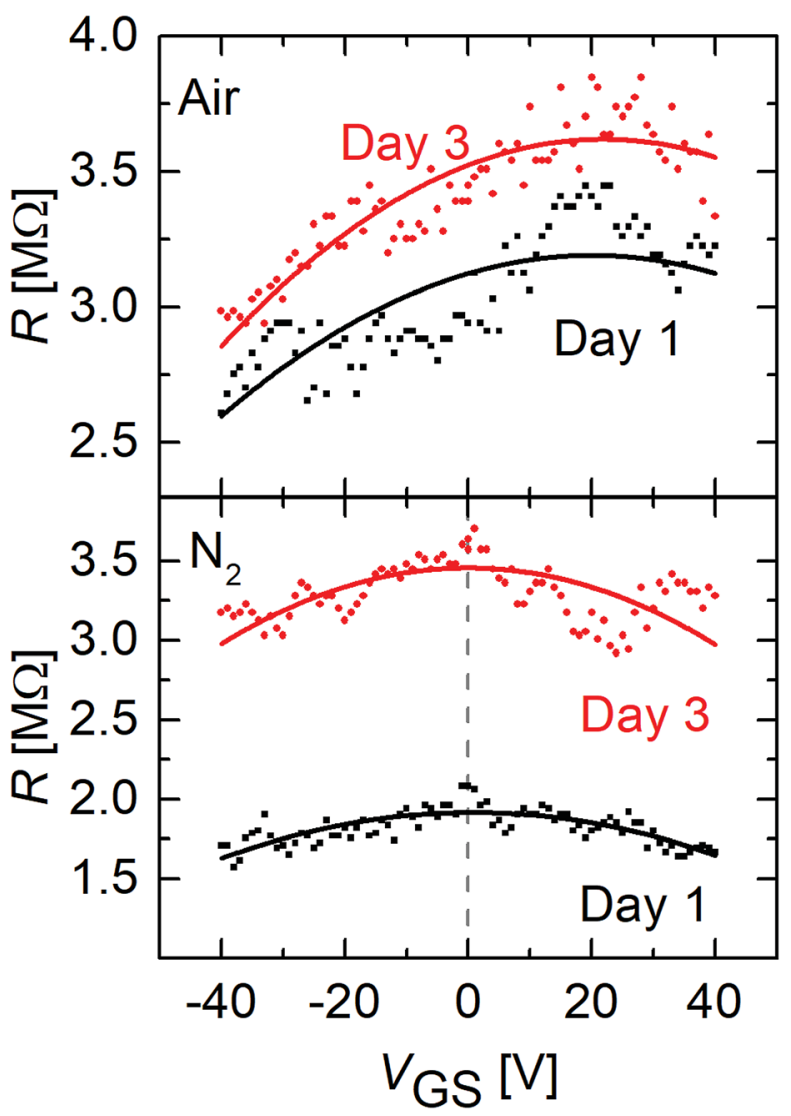

Fig. 5 Resistance of FGS with a C/O ratio of $x=26$ versus gate voltage for different days under air (top) and nitrogen (bottom) atmosphere. species such as water and oxygen into the FGSs. This view can be supported by the fact that FGSs with $x=60$ show the largest relative aging effect, as the material has been shown previously to be highly defective, while exhibiting only a comparably small number of oxygen-containing functional groups. ${ }^{41,42}$ Therefore, already a small amount of chemisorbing species can have a relatively large impact on the chemical structure and the electronic properties of the material.

\section{Conclusions}

In summary, we studied the electrical properties of single functionalized graphene sheets (FGSs) with varying amount of functional groups in a back-gated field-effect transistor device architecture. A reversible p-doping of FGSs as a result of exposure to ambient air is found for all $\mathrm{C} / \mathrm{O}$ ratios. This can be attributed to an adsorption of atmospheric species, which can be removed under nitrogen atmosphere. Further, a significant increase in the resistance in an 8 days' time period is seen, when the samples are exposed to laboratory air, which is more prominent for larger $\mathrm{C} / \mathrm{O}$ ratios. By determining separately the effect on the charge carrier density and the charge carrier mobility, we could distinguish between the (reversible) influence of adsorbed species from that of irreversible changes of the material due to chemisorption/oxidation. The latter one results in an alteration of the charge carrier mobility due to the chemisorbed oxygen containing atmospheric species, which serve as additional charge carrier scatterers.

The change of electrical properties over time has significant importance in applications of FGS (and rGO), where the material is exposed to potentially reactive chemical environments. It must be expected that material performance may change over time, as the graphene material becomes successively more oxidized. Besides exposure to air, also the contact with other materials such as polymer matrices may cause effects of the kind observed here.

\section{Conflicts of interest}

C. P. and I. A. A. declare financial interest in Vorbeck Materials Corp., a manufacturer of FGS-based materials and products.

\section{Acknowledgements}

We are gratefully thankful to Nicole Stracke, Maryan Barylyak and Piotr Sardyko for their experimental support. This work has 
been funded by the German Research Foundation (DFG) in the frame of the SPP 1459 "Graphene" under contract ME 1173/4-1.

\section{References}

1 K. S. Kim, Y. Zhao, H. Jang, S. Y. Lee, J. M. Kim, K. S. Kim, J.-H. Ahn, P. Kim, J.-Y. Choi and B. H. Hong, Nature, 2009, 457(7230), 706-710.

2 F. Schwierz, Nat. Nanotechnol., 2010, 5(7), 487-496.

3 X. Song, J. Hu and H. Zeng, J. Mater. Chem. C, 2013, 1(17), 2952.

4 Z. Yu, L. Tetard, L. Zhai and J. Thomas, Energy Environ. Sci., 2015, 8(3), 702-730.

5 F. Bonaccorso, L. Colombo, G. Yu, M. Stoller, V. Tozzini, A. C. Ferrari, R. S. Ruoff and V. Pellegrini, Science, 2015, 347(6217), 1246501.

6 J. D. Roy-Mayhew, D. J. Bozym, C. Punckt and I. A. Aksay, ACS Nano, 2010, 4(10), 6203-6211.

7 D. A. C. Brownson, D. K. Kampouris and C. E. Banks, J. Power Sources, 2011, 196(11), 4873-4885.

8 M. A. Pope, S. Korkut, C. Punckt and I. A. Aksay, J. Electrochem. Soc., 2013, 160(10), A1653-A1660.

9 S. Stankovich, D. A. Dikin, G. H. B. Dommett, K. M. Kohlhaas, E. J. Zimney, E. A. Stach, R. D. Piner, S. T. Nguyen and R. S. Ruoff, Nature, 2006, 442(7100), 282286.

10 T. Ramanathan, A. A. Abdala, S. Stankovich, D. A. Dikin, M. Herrera-Alonso, R. D. Piner, D. H. Adamson, H. C. Schniepp, X. Chen, R. S. Ruoff, S. T. Nguyen, I. A. Aksay, R. K. Prud'Homme and L. C. Brinson, Nat. Nanotechnol., 2008, 3(6), 327-331.

11 X. Huang, X. Qi, F. Boey and H. Zhang, Chem. Soc. Rev., 2012, 41(2), 666-686.

12 L. Gan, F. Qiu, Y.-B. Hao, K. Zhang, Z.-Y. Zhou, J.-B. Zeng and M. Wang, J. Mater. Sci., 2016, 51(11), 5185-5195.

13 F. Qiu, Y. Hao, X. Li, B. Wang and M. Wang, Composites, Part $B, 2015,71,175-183$.

14 H. Liu, Y. Li, K. Dai, G. Zheng, C. Liu, C. Shen, X. Yan, J. Guo and Z. Guo, J. Mater. Chem. C, 2016, 4(1), 157-166.

15 H. Liu, M. Dong, W. Huang, J. Gao, K. Dai, J. Guo, G. Zheng, C. Liu, C. Shen and Z. Guo, J. Mater. Chem. C, 2017, 5(1), 7383.

16 S. Stankovich, D. A. Dikin, R. D. Piner, K. A. Kohlhaas, A. Kleinhammes, Y. Jia, Y. Wu, S. T. Nguyen and R. S. Ruoff, Carbon, 2007, 45(7), 1558-1565.

17 M. J. McAllister, J.-L. Li, D. H. Adamson, H. C. Schniepp, A. A. Abdala, J. Liu, M. Herrera-Alonso, D. L. Milius, R. Car, R. K. Prud'homme and I. A. Aksay, Chem. Mater., 2007, 19(18), 4396-4404.

18 Y. Hernandez, V. Nicolosi, M. Lotya, F. M. Blighe, Z. Sun, S. De, I. T. McGovern, B. Holland, M. Byrne, Y. K. Gun'Ko, J. J. Boland, P. Niraj, G. Duesberg, S. Krishnamurthy, R. Goodhue, J. Hutchison, V. Scardaci, A. C. Ferrari and J. N. Coleman, Nat. Nanotechnol., 2008, 3(9), 563-568.

19 L. Yan, C. Punckt, I. A. Aksay, W. Mertin and G. Bacher, Nano Lett., 2011, 11(9), 3543-3549.
20 C. Punckt, F. Muckel, S. Wolff, I. A. Aksay, C. A. Chavarin, G. Bacher and W. Mertin, Appl. Phys. Lett., 2013, 102(2), 23114.

21 M. Zhou, Y. Wang, Y. Zhai, J. Zhai, W. Ren, F. Wang and S. Dong, Chem.-Eur. J., 2009, 15(25), 6116-6120.

22 S. Rumyantsev, G. Liu, W. Stillman, M. Shur and A. A. Balandin, J. Phys.: Condens. Matter, 2010, 22(39), 395302 .

23 M. Shur, S. Rumyantsev, G. Liu and A. A. Balandin, Proc. ICNF, 2011, vol. 21, pp. 145-149.

24 F. Yavari, C. Kritzinger, C. Gaire, L. Song, H. Gulapalli, T. Borca-Tasciuc, P. M. Ajayan and N. Koratkar, Small, 2010, 6(22), 2535-2538.

25 S. Ryu, L. Liu, S. Berciaud, Y.-J. Yu, H. Liu, P. Kim, G. W. Flynn and L. E. Brus, Nano Lett., 2010, 10(12), 49444951.

26 T. Pustelny, M. Setkiewicz, S. Drewniak, E. Maciak, A. Stolarczyk, M. Procek, M. Urbańczyk, K. Gut, Z. Opilski, I. Pasternak and W. Strupinski, Acta Phys. Pol., A, 2012, 122(5), 870-873.

27 W. Xu, T.-S. Lim, H.-K. Seo, S.-Y. Min, H. Cho, M.-H. Park, Y.-H. Kim and T.-W. Lee, Small, 2014, 10(10), 1999-2005.

28 A. Piazza, F. Giannazzo, G. Buscarino, G. Fisichella, A. La Magna, F. Roccaforte, M. Cannas, F. M. Gelardi and S. Agnello, J. Phys. Chem. C, 2015, 119(39), 22718-22723.

29 C. Melios, A. Centeno, A. Zurutuza, V. Panchal, C. E. Giusca, S. Spencer, S. R. P. Silva and O. Kazakova, Carbon, 2016, 103, 273-280.

30 C. Gómez-Navarro, R. T. Weitz, A. M. Bittner, M. Scolari, A. Mews, M. Burghard and K. Kern, Nano Lett., 2007, 7(11), 3499-3503.

31 I. Jung, D. A. Dikin, R. D. Piner and R. S. Ruoff, Nano Lett., 2008, 8(12), 4283-4287.

32 C. W. Lee, B. J. Min, S. I. Kim and H. K. Jeong, Carbon, 2013, 54, 353-358.

33 B. Gupta, N. Kumar, K. A. Titovich, K. V. Ivanovich, S. A. Vyacheslavovich and S. Dash, Surf. Interfaces, 2017, 7, 6-13.

34 D. K. Schroder, Semiconductor Material and Device Characterization, John Wiley \& Sons, Inc, Hoboken, NJ, USA, 1998.

35 S. Kim, I. Jo, D. C. Dillen, D. A. Ferrer, B. Fallahazad, Z. Yao, S. K. Banerjee and E. Tutuc, Phys. Rev. Lett., 2012, 108(11), 116404.

36 H. Zhong, Z. Zhang, H. Xu, C. Qiu and L.-M. Peng, AIP Adv., 2015, 5(5), 57136.

37 A. Das, S. Pisana, B. Chakraborty, S. Piscanec, S. K. Saha, U. V. Waghmare, K. S. Novoselov, H. R. Krishnamurthy, A. K. Geim, A. C. Ferrari and A. K. Sood, Nat. Nanotechnol., 2008, 3(4), 210-215.

38 C. Mattevi, G. Eda, S. Agnoli, S. Miller, K. A. Mkhoyan, O. Celik, D. Mastrogiovanni, G. Granozzi, E. Garfunkel and M. Chhowalla, Adv. Funct. Mater., 2009, 19(16), 2577-2583.

39 A. K. Geim and K. S. Novoselov, Nat. Mater., 2007, 6(3), 183191. 
40 A. Liscio, G. P. Veronese, E. Treossi, F. Suriano, F. Rossella, V. Bellani, R. Rizzoli, P. Samorì and V. Palermo, J. Mater. Chem., 2011, 21(9), 2924.
41 M. A. Pope and I. A. Aksay, J. Phys. Chem. C, 2015, 119(35), 20369-20378.

42 J. D. Roy-Mayhew, M. A. Pope, C. Punckt and I. A. Aksay, ACS Appl. Mater. Interfaces, 2016, 8(14), 9134-9141. 